
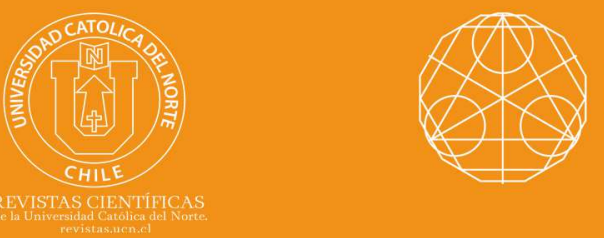

\title{
A new approach for Volterra functional integral equations with non-vanishing delays and fractional Bagley-Torvik equation
}

Fateme Ghomanjani' ${ }^{1}$ orcid.org/0000-0002-5319-9389

Kashmar Higher Education Institute, Dept. of Mathematics, Kashmar, Iran.

$1 \square$ fatemeghomanjani@gmail.com

Received: 2020/02/20| Accepted: 2020/07/20

\section{Abstract:}

A numerical technique for Volterra functional integral equations (VFIEs) with non-vanishing delays and fractional Bagley-Torvik equation is displayed in this work. The technique depends on Bernstein polynomial approximation. Numerical examples are utilized to evaluate the accurate results. The findings for examples figs and tables show that the technique is accurate and simple to use.

Keywords: Integral equations; Numerical solution; Fractional BagleyTorvik equation.

MSC (2020): 65K10, 26A33, 49K15.

\section{Cite this article as (IEEE citation style):}

F. Ghomanjani, "A new approach for Volterra functional integral equations with non-vanishing delays and fractional Bagley-Torvik equation", Proyecciones (Antofagasta, On line), vol. 40, no. 4, pp. 885-903, 2021, doi: 10.22199/issn.0717-6279-4010

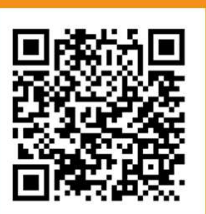

Article copyright: (C) 2021 Fateme Ghomanjani. This is an open access article distributed under the terms of the Creative Commons License, which permits unrestricted use and distribution provided the original author and source are credited. 


\section{Introduction}

The following general Volterra functional integral equation (VFIE) is considered

$$
\begin{aligned}
y(t) & =g(t)+b(t) y(\theta(t))+\int_{t_{0}}^{t} k_{1}(t, s) y(s) d s \\
& +\int_{t_{0}}^{\theta(t)} k_{2}(t, s) y(s) d s, \quad t \in I:=\left(t_{0}, T\right]=\left(t_{0}, t_{f}\right], \\
y(t) & =\phi(t), \quad t \in I_{\theta}:=\left[\theta\left(t_{0}\right), t_{0}\right], t_{f}=T,
\end{aligned}
$$

where $0<q<1(\theta(t)$ is the function which related to $q)$, and $k_{1}(t, s)$ and $k_{2}(t, s)$ are assumed to be continuous functions on their respective domains $D:=\left\{(t, s): t_{0} \leq s \leq t \leq T, t \in I\right\}$ and $D_{\theta}:=\left\{(t, s): \theta\left(t_{0}\right) \leq s \leq \theta(t), t \in\right.$ $I\}$ (for more explanation see [1]).

The $h p$ variant of the discontinuous Galerkin technique for the numerical solution of delay differential equations (DDEs) with nonlinear vanishing delays was introduced in [2]. In [3], the existence, uniqueness of solutions for VFIEs with the time delay were analyzed. Huang et al. [4] studied the superconvergence of the discontinuous Galerkin solutions for DDEs with proportional vanishing at $t=0$.

Additionally many papers manage the Bezier curves. In [5] and [6], authors utilized the Bezier curves in approximating functions. For solving differential equations (DEs) numerically, authors in [7] proposed the utilization of Bezier curves. Also, to solve delay differential equation, the Bezier control points strategy is utilized (see [8]). Some other uses of the Bezier functions are found in (see [9]). In the present work, we use the proposed method in [9] for solving VFIE.

The outline of this sequel is as follow: In Section 2, problem statement is stated. Explanation of the problem is explained in Section 3. Also, error estimation based on residual error function is presented in Section 4. Some numerical results are provided in Section 5. Also, a remark is stated about fractional Bagley-Torvik equation. Finally, Section 6 will give a conclusion briefly. 


\section{Problem Statement}

For finding an approximate solution of Eq. (1.1), one may consider the following $y(t)$ :

$$
y(t) \cong y_{n}(t)=\sum_{i=0}^{n} c_{i} B_{i, n}(t), t_{0} \leq t \leq t_{f}, n \geq 1
$$

where

$$
\begin{aligned}
& B_{r, n}\left(\frac{t-t_{0}}{h}\right)=\left(\begin{array}{c}
n \\
r
\end{array}\right) \frac{1}{h^{n}}\left(t_{f}-t\right)^{n-r}\left(t-t_{0}\right)^{r}, \quad t_{0} \leq t \leq t_{f}, \quad 0 \leq i \leq n, \\
& h=t_{f}-t_{0}
\end{aligned}
$$

and $c_{i}, i=0,1, \ldots, n$ are the unknown control points. In this paper, one may utilize Bezier curve for solving VFIE. This technique is applied in [10], [11] for solving optimal control of problems (OCPs) and some linear OCPs with pantograph delays. The convergence of the proposed technique is proven in this sequel.

By substituting $y(t)$ in (1.1), one may define

$$
f_{\text {objective }}=\sum_{i=0}^{n} c_{i}^{2}, \quad t \in\left[t_{0}, t_{f}\right]
$$

Now, our goal is to solve the following problem to find the values $c_{r}$, for $r=0,1, \ldots, n$.

$$
\begin{array}{ll}
\min & f_{\text {objective }} \\
\text { s.t. } & y(t)=g(t)+b(t) y(\theta(t))+\int_{t_{0}}^{t} k_{1}(t, s) y(s) d s \\
& +\int_{t_{0}}^{\theta(t)} k_{2}(t, s) y(s) d s, \quad t \in I:=\left(t_{0}, T\right], \\
& y(t)=\phi(t), \quad t \in I_{\theta}:=\left[\theta\left(t_{0}\right), t_{0}\right],
\end{array}
$$

where "s.t." is abbreviation of "such that". 


\section{Explanation of the problem}

Now, the following problem is considered

$$
\begin{aligned}
(D y)(t) & =y(t)-g(t)+b(t) y(\theta(t)) \\
& +\int_{0}^{t} k_{1}(t, s) y(s) d s+\int_{0}^{\theta(t)} k_{2}(t, s) y(s) d s, \\
y_{n}(t) & =\sum_{i=0}^{n} c_{i} B_{i, n}(t), \quad 0 \leq t \leq t_{f}, \\
y(t) & =\phi(t), t \leq 0 .
\end{aligned}
$$

Without loss of generality, one may consider $t_{0}=0$. Firstly, we have

$$
\begin{aligned}
\left(D y_{n}\right)(t) & =\sum_{i=0}^{n} c_{i} B_{i, n}(t)-g(t) \\
& +b(t) \sum_{i=0}^{n} c_{i} B_{i, n}(\theta(t)) \\
& +\int_{0}^{t} k_{1}(t, s) \sum_{i=0}^{n} c_{i} B_{i, n}(s) d s \\
& +\int_{0}^{\theta(t)} k_{2}(t, s) \sum_{i=0}^{n} c_{i} B_{i, n}(s) d s \\
& =\sum_{i=0}^{n} c_{i}\left(B_{i, n}(t)+b(t) B_{i, n}(\theta(t))\right. \\
& +\int_{0}^{t} k_{1}(t, s) B_{i, n}(s) d s \\
& \left.+\int_{0}^{\theta(t)} k_{2}(t, s) B_{i, n}(s) d s\right) \\
& -g(t) \\
& =\sum_{i=0}^{n} c_{i} \alpha_{i}(t)-g(t),
\end{aligned}
$$

where $\alpha_{i}=\left(B_{i, n}(t)+b(t) B_{i, n}(\theta(t))\right.$ 
$\left.+\int_{0}^{t} k_{1}(t, s) B_{i, n}(s) d s+\int_{0}^{\theta(t)} k_{2}(t, s) B_{i, n}(s) d s\right), i=0,1,2, \ldots, n$. Now, the

following real function is defined

$$
J=J\left(c_{0}, c_{1}, \ldots, c_{n}\right)=\int_{0}^{t_{f}}\left(D y_{n}\right)^{2}(t) d t
$$

according to the above equations, we have

$$
\begin{aligned}
& \min J(C) \\
& \text { s.t. } \quad I(C)=\sum_{i=0}^{n} c_{i}\left(\sum_{k=0}^{n} c_{k} B_{k, n}\left(-\frac{j}{n}\right)\right)-\phi\left(-\frac{j}{n}\right), \\
& j=0,1, \ldots, n,
\end{aligned}
$$

where $C=\left(c_{0}, c_{1}, \ldots, c_{n}\right)$. There are various techniques for solving this problem, such as the Newton's method, the gradient descent, the conjugategradient technique and the Lagrange technique. Here, the Lagrange-multiplier method is considered to solve the problem (3.1). By the Lagrange function, one may have the following form of

$$
L=J(C)+\mu I(C),
$$

Hence

$$
\begin{aligned}
\frac{\partial L}{\partial c_{i}} & =2 \int_{0}^{t_{f}}\left(D y_{n}\right)(t) \frac{\partial\left(D y_{n}\right)(t)}{\partial c_{i}} d t+\mu \frac{\partial I(C)}{\partial c_{i}} \\
& =2\left(\sum_{j=0}^{n} c_{j} \int_{0}^{t_{f}} \alpha_{j}(t) \alpha_{i}(t) d t\right. \\
& \left.-\int_{0}^{t_{f}} g(t) \alpha_{i}(t) d t\right) \\
& +\mu \sum_{k=0}^{n} c_{k} B_{k, n}\left(-\frac{i}{n}\right), \\
\frac{\partial L}{\partial \mu} & =I(C),
\end{aligned}
$$


As we known, a necessary condition (3.1) is that

$$
\left\{\begin{array}{l}
\frac{\partial L}{\partial c_{i}}=0, \quad 0 \leq i \leq n, \\
\frac{\partial L}{\partial \mu}=0
\end{array}\right.
$$

For simplification, one may define

$$
(f, g)=\int_{0}^{t_{f}} f(t) g(t) d t
$$

Combining Eqs. (3.2)-(3.4), we have

$$
\begin{aligned}
& \sum_{j=0}^{n} 2 c_{j}\left(\alpha_{j}, \alpha_{i}\right)+\mu \sum_{k=0}^{n} c_{k} B_{k, n}\left(-\frac{i}{n}\right)=2\left(g, \alpha_{i}\right), \\
& 0 \leq i \leq n, \\
& \sum_{i=0}^{n} c_{i} B_{i, n}(t)=\phi(t), \quad t \leq 0,
\end{aligned}
$$

we can rewrite Eq. (3.5) as the following form

$$
\sum_{i=0}^{n} c_{i} B_{i, n}\left(-\frac{j}{n}\right)=\phi\left(-\frac{j}{n}\right), \quad-n \leq j \leq 0
$$

Now, we write

$$
\begin{aligned}
G \tilde{C} & =F \\
G & =\left[\begin{array}{ccccc}
2\left(\alpha_{0}, \alpha_{0}\right) & 2\left(\alpha_{1}, \alpha_{0}\right) & \ldots & 2\left(\alpha_{n}, \alpha_{0}\right) & \phi(0) \\
2\left(\alpha_{0}, \alpha_{1}\right) & 2\left(\alpha_{1}, \alpha_{1}\right) & \ldots & 2\left(\alpha_{n}, \alpha_{1}\right) & \phi\left(-\frac{1}{n}\right) \\
\vdots & \vdots & \vdots & \vdots & \vdots \\
2\left(\alpha_{0}, \alpha_{n}\right) & 2\left(\alpha_{1}, \alpha_{n}\right) & \ldots & 2\left(\alpha_{n}, \alpha_{n}\right) & \phi\left(-\frac{n-1}{n}\right) \\
B_{0, n}(0) & B_{1, n}(0) & \ldots & B_{n, n}(0) &
\end{array}\right], \\
\tilde{C} & =\left[\begin{array}{cc}
\left.c_{0}, c_{1}, \ldots, c_{n}, \mu\right]^{T}, \\
F & =\left[2\left(g, \alpha_{0}\right), \ldots, 2\left(g, \alpha_{n}\right), \phi(0)\right.
\end{array}\right]^{T} .
\end{aligned}
$$

the unique solution of linear system (3.6) is $y_{n}(t)=\sum_{i=0}^{n} c_{i} B_{i, n}(t)$ is called an optimal control approximation solution (OCAS). If $\lim _{n \rightarrow \infty} \int_{0}^{t_{f}}\left(D y_{n}\right)^{2}(t) d t=$ 0 then the OCAS converges to the exact solution. 


\section{Error estimation based on residual error function}

Now, some theorems are stated for the error estimation.

Theorem 4.1. Let $y(t)$ is a continuous exact solution defined on $\left[0, t_{f}\right]$, and $y_{n}(t)$ is an OCAS of this problem. If there exists $P_{n}(t)=\sum_{i=0}^{n} c_{i} B_{i, n}(t)$, $c_{i} \in R$ such that for any $t \in\left[0, t_{f}\right], \lim _{n \rightarrow \infty} P_{n}(t)=y(t)$, then $\lim _{n \rightarrow \infty} \int_{0}^{t_{f}}\left(D y_{n}\right)^{2}(t) d t=0$.

Proof. By the Weierstrass theorem it follows that there exists the sequence of polynomials, $P_{n}(t)=\sum_{i=0}^{n} c_{i} B_{i, n}(t)$ such that $\lim _{n \rightarrow \infty} P_{n}(t)=y(t)$, that is

$$
\lim _{n \rightarrow \infty} \int_{0}^{t_{f}}\left(D P_{n}\right)^{2}(t) d t=0
$$

Hence, one may have

$$
\begin{array}{r}
0 \leq \int_{0}^{t_{f}}\left(D y_{n}\right)^{2}(t) d t \leq \int_{0}^{t_{f}}\left(D P_{n}\right)^{2}((t) d t, \\
0 \leq \lim _{n \rightarrow \infty} \int_{0}^{t_{f}}\left(D y_{n}\right)^{2}(t) d t \leq \lim _{n \rightarrow \infty} \int_{0}^{t_{f}}\left(D P_{n}\right)^{2}(t) d t,
\end{array}
$$

By Eqs.(4.1) and (4.3), the proof is completed.

Now, the approximate solution obtained by this technique can be stated as:

$$
y_{n}(t)=\sum_{i=0}^{n} c_{i} B_{i, n}(t)
$$

Presently, in the following theorem, an upper bound for the error estimation of the proposed technique can be proven.

Theorem 4.2. Let $y(t) \in C^{n+1}\left[0, t_{f}\right]$ is the exact solution of explained problem, $y_{n}(t)=\sum_{i=0}^{n} c_{i} B_{i, n}(t)$ is the solution with degree $n$, hence

$$
\begin{aligned}
& \left\|y(t)-y_{n}(t)\right\|_{\infty} \leq \frac{M}{(n+1) !} \max _{i=0,1, \ldots, n}\left|\tilde{c_{i}}\right|, \\
& M=\max _{0 \leq t \leq t_{f}}\left|y^{n+1)}(t)\right|, \tilde{c_{k}}=\frac{y^{(k)}(0)}{k !}-c_{i},
\end{aligned}
$$


Proof. See [12].

Here, the residual error function of the numerical solution is defined $y_{n}(t)$ as

$$
R_{n}(t)=L\left\{y_{n}(t)\right\}-g(t)
$$

and

$$
e_{n}(t)=y(t)-y_{n}(t)
$$

where $y(t)$ is exact solution. Hence

$$
\begin{aligned}
& L\left\{e_{n}(t)\right\}=L\{y(t)\}-L\left\{y_{n}(t)\right\}=-R_{n}(t), \\
& \sum_{i=0}^{n} c_{i} B_{i, n}(t)=\phi(t), \quad t \leq 0 .
\end{aligned}
$$

\section{Numerical applications}

In this section, some results are given to demonstrate the quality of the sated technique in approximating the solution of delay Volterra integral equations (1.1).

Example 1. Now, the following proportional delay VFIE is considered

$$
\begin{aligned}
y(t) & =\left(e^{t}+e^{1-t}-e-1\right)+y(q t)+\int_{1}^{t} e^{-t} y(s) d s \\
& -\int_{1}^{q t} y(s) d s, \quad t \in[0,3], \\
y(t) & =e^{t}, t \leq 1,
\end{aligned}
$$

where $y_{\text {exact }}(t)=e^{t}$. One may obtain $y(t)=0.04102190678 t^{5}-0.0987753295 t^{4}+$ $0.4132317318 t^{3}+0.3108745122 t^{2}+1.055479444 t+0.9964495623$ with this technique by $n=5$. The approximate solution for $y(t)$ is shown in Fig. 1 . 


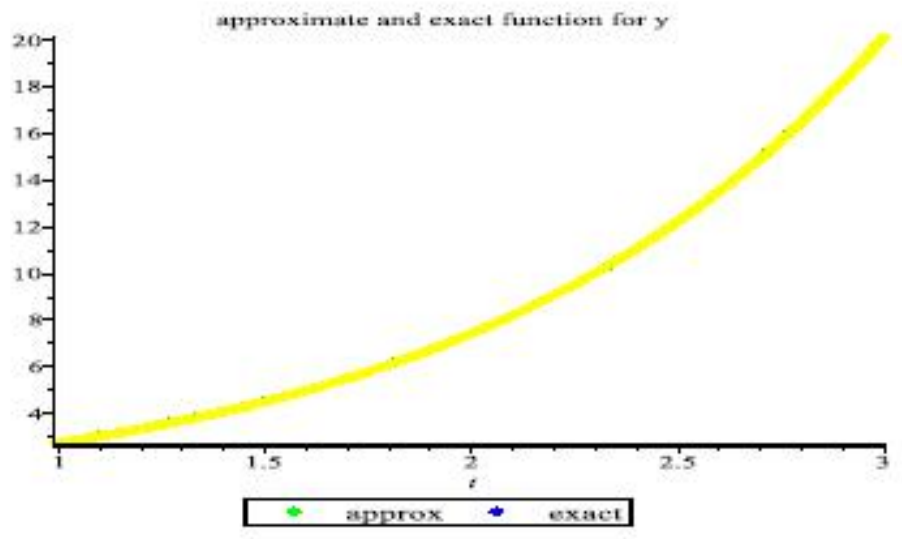

Example 2. The following vanishing delay Volterra integral equation (VIE) is considered (see [13])

$$
y(t)=\frac{1}{2}\left(1+e^{-q t}\right)-\int_{0}^{t} y(s) d s+\frac{1}{2} \int_{0}^{q t} y(s) d s,
$$

where $q=0.2$, and $y_{\text {exact }}(t)=e^{-t}$. One may obtain $y(t)=1-0.99941777 t+$ $0.4953779080 t^{2}-0.15359822 t^{3}+0.0255175232 t^{4}$ with this method by $n=4$. The approximate solution for $y(t)$ is shown in Fig. 2. Table 1 demonstrates the errors of the this technique where $\|$ error $\|_{\infty}$ in [13] and this paper are respectively $2 \times 10^{-4}$ (for $n=4, m=1$ ) and 0.0 .

Table 1: The errors of the this method for Example 2

\begin{tabular}{cc}
\hline$t$ & error of $y$ \\
\hline 0.1 & 0.00002353764412 \\
0.2 & 0.0000128515059 \\
0.3 & $7.4 \times 10^{-11}$ \\
0.4 & 0.000003726205 \\
0.5 & $8 \times 10^{-11}$ \\
0.6 & 0.00000360418 \\
0.7 & $3 \times 10^{-11}$ \\
0.8 & 0.0000116301 \\
0.9 & 0.0000206027 \\
1.0 & 0.0 \\
\hline
\end{tabular}


Example 3. The following vanishing delay VIE is considered (see [13])

$$
\begin{aligned}
& y(t)=f(t)+\int_{0}^{t} y(s) d s+\int_{0}^{q t} e^{-t} y(s) d s, \\
& f(t)=\cos (t)-\sin (t)-e^{-t} \sin (0.5 t),
\end{aligned}
$$

where $q=0.5$, and exact solution is $y(t)=\cos (t)$. One may obtain $y(t)=$ $1+0.000271524 t-0.502368133 t^{2}+0.006222416 t^{3}+0.03617649887 t^{4}$ with this technique by $n=4$. The approximate solution for $y(t)$ is shown in Fig. 3. Table 2 demonstrates the errors of the this technique.
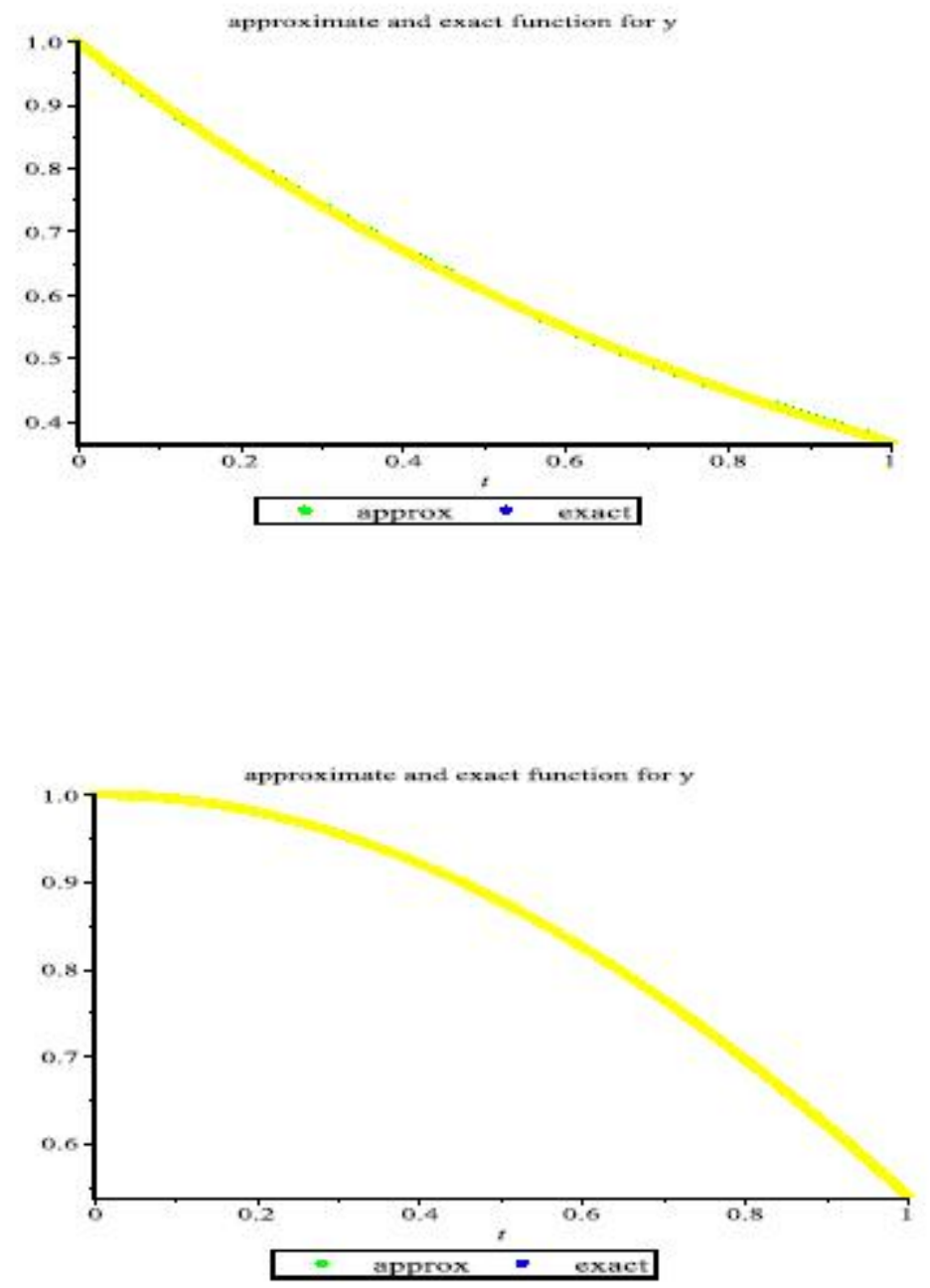
Remark 5.1. The fractional differential equations have drawn expanding consideration as a result of their vital applications in science, material science, and designing (see $[14,15,16,17,18,19,20])$. Generally, the solving of most fractional differential equations are definitely not simple. The Bagley-Torvik equation was introduced by the authors of [21]. In different papers, it has been examined. This equation has been concentrated both diagnostically and, numerically in [22]. These techniques included expansion formula for fractional derivative [23], Haar wavelet, pseudo-spectral scheme [24], Bessel collocation technique [25], Taylor collocation method [26]. Mekkaoui and Hammouch [27] solved the Bagley-Torik equation by the fractional iteration method (FIM). In addition, the stability of the Bagley-Torvik equation is given [28]. Mashayekhi and Razzaghi [29] stated a new numerical tech- nique by utilizing hybrid functions approximation for solving the fractional Bagley-Torvik equation.

In this remark, one may utilize Bezier curves technique for solving fractional Bagley-Torvik equation.

\subsection{Basic Preliminaries}

Definition 5.1. The caputo's fractional derivative of order $\alpha$ is stated in [25]

$$
\begin{aligned}
D^{\alpha} f(t) & =\overline{\Gamma\left(n_{1}-\alpha\right)} \int_{0}^{t}(t-s)^{-\alpha-1+n_{1}} f^{\left(n_{1}\right)}(s) d s, \\
n_{1}-1 & \leq \alpha \leq n_{1}, n_{1} \in \mathbf{N},
\end{aligned}
$$

where $\alpha>0$ and $n_{1}$ is the smallest integer greater than $\alpha$.

Table 2: The errors of the this method for Example 3

\begin{tabular}{cc}
\hline$t$ & error of $y$ \\
\hline 0.1 & $0.91459 \times 10^{-5}$ \\
0.2 & $6.634 \times 10^{-7}$ \\
0.3 & 0.000007129 \\
0.4 & $0.69327 \times 10^{-5}$ \\
0.5 & $1.000000000 \times 10^{-10}$ \\
0.6 & 0.0000072877 \\
0.7 & 0.0000077604 \\
0.8 & 0.0000013243 \\
0.9 & 0.0000122423 \\
\hline
\end{tabular}




\subsection{Function approximation}

Utilizing Bezier curves, this technique is to approximate the solutions $f(t)$ where $f(t)$ is given in Eq. (5.1). Define the Bezier polynomials of degree $n$ that approximate over the interval $t \in\left[t_{0}, t_{f}\right]$ as follows:

$$
f \approx P^{n} f=\sum_{r=0}^{n} c_{r} B_{r, n}\left(\frac{t-t_{0}}{h}\right)=C^{T} B(t),
$$

where $h=t_{f}-t_{0}, C^{T}=\left[c_{0}, c_{1}, \ldots, c_{n}\right]^{T}$,

$$
\begin{aligned}
& B^{T}(t)=\left[B_{0, n}(t), B_{1, n}(t), \ldots, B_{n, n}(t)\right]^{T}, \\
& B_{r, n}\left(\frac{t-t_{0}}{h}\right)=\left(\begin{array}{l}
n \\
r
\end{array}\right) \frac{1}{h^{n}}\left(t_{f}-t\right)^{n-r}\left(t-t_{0}\right)^{r},
\end{aligned}
$$

is the Bernstein polynomial with degree $n$ for $t \in\left[t_{0}, t_{f}\right]$, and $c_{r}$ is the control point [9].

\subsection{Error bound for the Bezier curve}

The error bound is presented for the Bezier curve now.

Theorem 5.1. Let $f \in H^{\mu}(0,1)$ with $\mu \geq 0$, then one may have

$$
\left\|f-P^{n} f\right\|_{L^{2}(0,1)} \leq C M^{-\mu}|f|_{H^{0 ; \mu ; M ; n}(0,1)},
$$

where

$$
\begin{aligned}
& H^{\mu}(0,1)=W^{\mu, 2}(0,1)= \\
& \left\{f \in L^{2}(0,1)\left|\partial^{\alpha} f \in L^{2}(0,1),\right| \alpha \mid \leq \mu\right\}
\end{aligned}
$$

Proof. See [29].

Now, one may derive the operator $I^{\alpha}$ for $B(t)$ in Eq. 11 given by

$$
I^{\alpha} B(t)=\bar{B}(t, \alpha),
$$

where

$$
\bar{B}(t, \alpha)=\left[I^{\alpha} B_{0, n}(t), \ldots, I^{\alpha} B_{n, n}(t)\right]^{T} .
$$




\subsection{Problem statement}

One may introduce the following Bagley-Torvik fractional equation

$$
A D^{(2)} f(t)+B D^{\left(\frac{3}{2}\right)} f(t)+C f(t)=g(t) .
$$

In this sequel, one may have the following problems:

\subsubsection{Problem (a)}

Bagley-Torvik equations in Eq. 22 with the conditions given by:

$$
f(0)=f_{0}, f^{\prime}(0)=f_{0}^{\prime},
$$

where $f_{0}$ and $f_{0}^{\prime}$ are given.

\subsubsection{Problem (b)}

Bagley-Torvik equations in Eq. 22 with the following conditions

$$
f(0)=f_{0}, f(1)=f_{1},
$$

where $f_{0}$ and $f_{1}$ are given.

For more explanation about these problems, one can study [29].

\subsection{Error bound for problem (a)}

Now, the error bound $E^{n}$ is found for problem (a).

Theorem 5.2. Let $f \in H^{\mu}(0,1)$ with $\mu \geq 0$, we have

$$
\begin{aligned}
\left\|E_{n}\right\|_{L^{2}(0,1)} & \leq\left(c M^{-\mu} n^{-\mu}\left\|f^{(\mu)}\right\|_{L^{2}(0,1)}\right) \\
& \times\left(A+\frac{B}{\Gamma\left(\frac{3}{2}\right)}+\frac{C}{\Gamma(3)}\right) .
\end{aligned}
$$

Proof. See [29]. 


\subsection{Numerical applications}

Now, for demonstrating the applicability and accuracy of this technique some examples are solved. The package of Maple version 14 has been used to solve the test problems.

Example 4. Now, one may consider Eqs. 22 and 24 such that (see [29])

$$
\begin{aligned}
& A=1, B=\frac{8}{17}, C=\frac{13}{51}, f_{0}=0, f_{1}=0, \\
& g(t)=\frac{t^{-\frac{1}{2}}}{89250 \sqrt{\pi}}(48 p(t)+7 \sqrt{t} q(t))
\end{aligned}
$$

where

$$
\begin{aligned}
& p(t)=16000 t^{4}-32480 t^{3}+2128 t^{2}-4746 t+189 \\
& q(t)=3250 t^{5}-9425 t^{4}+264880 t^{3}-44 .
\end{aligned}
$$

the exact solution is $f(t)=t^{5}-\frac{29 t^{4}}{10}+\frac{76 t^{3}}{25}-\frac{339 t^{2}}{250}+\frac{27 t}{125}$.

With $n=5$, the obtained $f(t)$ is $0.216 t(1-t)^{4}-0.492 t^{2}(1-t)^{3}+0.2680000000 t^{3}(1-$ $t)^{2}-0.024 t^{4}(1-t)$ with $c_{0}=0, c_{1}=0.0432000000000003, c_{2}=-0.0492000000000008$, $c_{3}=0.0268000000000010, c_{4}=-0.00480000000000036$, and $c_{5}=0$. The obtained error is zero (see Table 3). The graphs of approximated and exact solution $f(t)$ are plotted in Fig. 4.

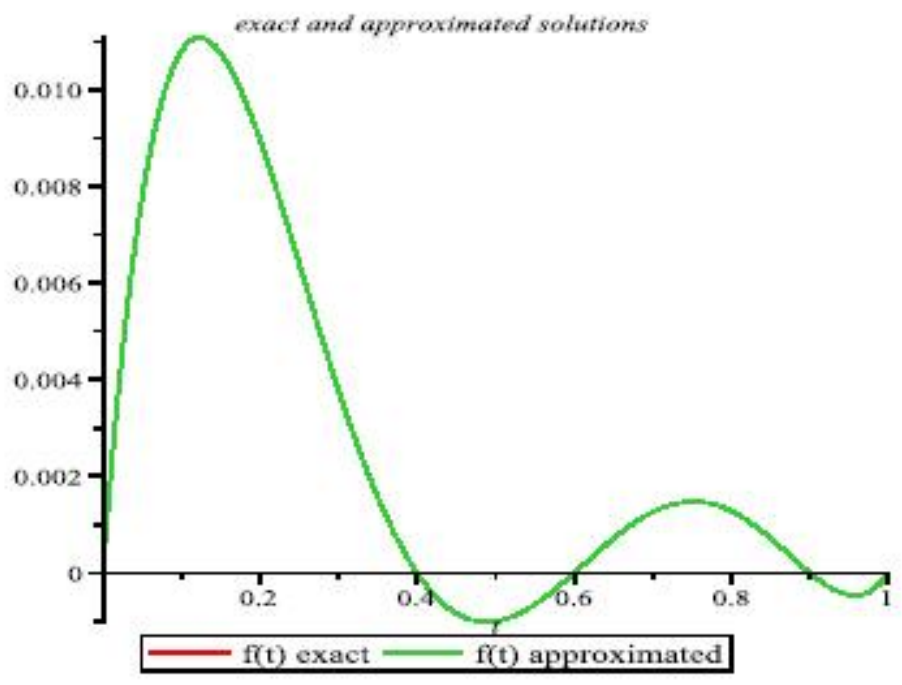


Table 3: The errors of the this method for Example 4

\begin{tabular}{cc}
\hline$t$ & error of $f$ \\
\hline 0.1 & 0.0 \\
0.2 & 0.0 \\
0.3 & 0.0 \\
0.4 & 0.0 \\
0.5 & 0.0 \\
0.6 & 0.0 \\
0.7 & 0.0 \\
0.8 & 0.0 \\
0.9 & 0.0 \\
\hline
\end{tabular}

Example 5. One may consider Eqs. 22 and 24 such that (see [29])

$$
\begin{aligned}
& f(0)=0, f(1)=0, g(t)=\frac{4 \sqrt{t}}{\sqrt{\pi}}+t(t-1), \\
& A=0, B=C=1,
\end{aligned}
$$

where the exact solution is $f(t)=t^{2}-t$.

With $n=5$, the obtained $f(t)$ is $-t(1-t)^{4}-3 t^{2}(1-t)^{3}-3 t^{3}(1-t)^{2}-1 t^{4}(1-t)$ with $c_{0}=0, c_{1}=-0.200000000000002, c_{2}=-0.299999999999996, c_{3}=$ $-0.300000000000004, c_{4}=-0.19999999999999$, and $c_{5}=0$. The obtained error is zero (see Table 4). The graphs of approximated and exact solution $f(t)$ are plotted in Fig. 5.

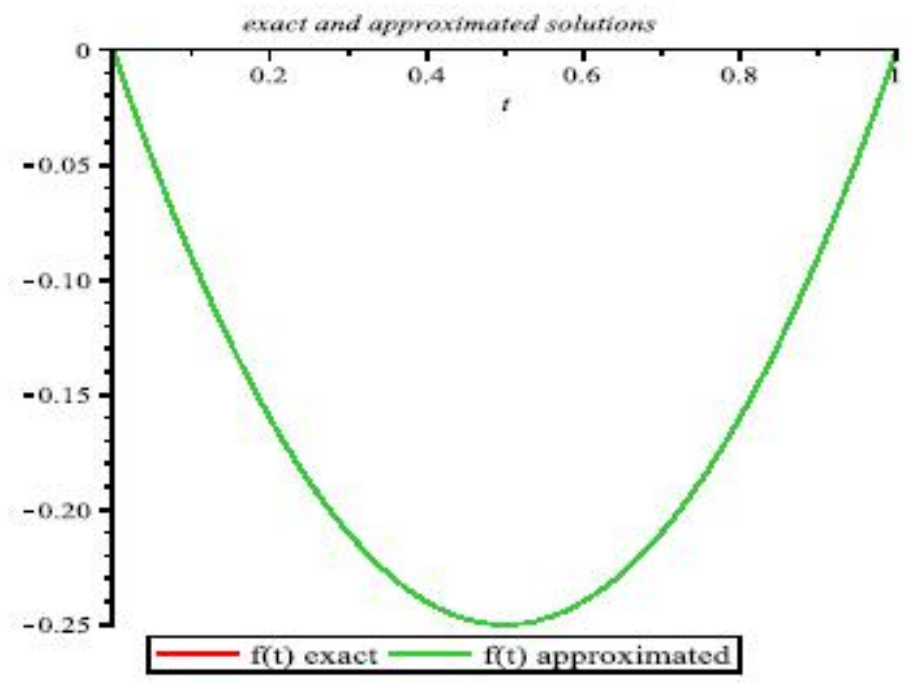


Table 4: The errors of the this method for Example 5

\begin{tabular}{cc}
\hline$t$ & error of $f$ \\
\hline 0.1 & 0.0 \\
0.2 & 0.0 \\
0.3 & 0.0 \\
0.4 & 0.0 \\
0.5 & 0.0 \\
0.6 & 0.0 \\
0.7 & 0.0 \\
0.8 & 0.0 \\
0.9 & 0.0 \\
\hline
\end{tabular}

\section{Conclusions}

The aim of this sequel is to improve an effective and accurate technique for solving VFIE and fractional Bagley-Torvik equation. The Bezier curve strategy is utilized to obtain the approximate solution of this problem.

Some results are included to explain the validity of this technique.

\section{References}

[1] W. Ming and C. Huang, "Collocation methods for Volterra functional integral equations with non-vanishing delays", Applied mathematics and computation, vol. 296, pp. 198-214, 2017, doi: 10.1016/j.amc. 2016.10.021

[2] Q. Huang, H. Xie, and H. Brunner, "The \$hp\$ discontinuous Galerkin method for delay differential equations with nonlinear Vanishing delay", SIAM journal on scientific computing, vol. 35, no. 3, pp. A1604-A1620, 2013, doi:10.1137/ 120901416

[3] H. Xie, R. Zhang, and H. Brunner, "Collocation methods for general Volterra functional integral equations with vanishing delays", SIAM journal on scientific computing, vol. 33, no. 6, pp. 3303-3332, 2011, doi: $10.1137 / 100818595$

[4] Q. Huang, H. Xie, and H. Brunner, "Super convergence of a discontinuous Galerkin solutions for delay differential equations of pantograph type", SIAM journal on scientific computing, vol. 33, no. 5, pp. 2664-2684, 2011, doi: 10.1137/ 110824632 
[5] K. Harada and E. Nakamae, "Application of the Bézier curve to data interpolation", Computer-aided design, vol. 14, no. 1, pp. 55-59, 1982, doi: 10.1016/ 0010-4485(82)90013-6

[6] G. Nürnberger and F. Zeilfelder, "Developments in bivariate spline interpolation", Journal of computational and applied mathematics, vol. 121, no. 1-2, pp. 125-152, 2000, doi:10.1016/ S0377-0427(00)00346-0

[7] J. Zheng, T. W. Sederberg, and R. W. Johnson, "Least squares methods for solving differential equations using Bézier control points", Applied numerical mathematics, vol. 48, no. 2, pp. 237-252, 2004, doi: 10.1016/j.apnum.2002.01.001

[8] F. Ghomanjani and M. H. Farahi, "The Bézier control points method for solving delay differential equation", Intelligent control and automation, vol. 3, no. 2, pp. 188-196, 2012, doi: 10.4236/ ica.2012.32021

[9] F. Ghomanjani, M. H. Farahi, and M. Gachpazan, "Bézier control points method to solve constrained quadratic optimal control of time varying linear systems", Computational and applied mathematics, vol. 31, no. 3, pp. 433-456, 2012, doi: 10.1590/ S1807-03022012000300001

[10] F. Ghomanjani and M. H. Farahi, "Optimal control of switched systems based on Bézier control points", International journal of intelligent systems and applications, vol. 4, no. 7, pp. 16-22, 2012, doi: 10.5815/ ijisa.2012.07.02

[11] F. Ghomanjani, M H. Farahi, and A. V. Kamyad, "Numerical solution of some linear optimal control systems with pantograph delays", IMA journal of mathematical control and information, vol. 32, no. 2, pp. 225-243. 2015, doi: 10.1093/imamci/ dnt037

[12] L. J. Xie, C. L. Zhou, and S. Xu, "An effective numerical method to solvea class of nonlinear singular boundary value problems using improved differential transform method", SpringerPlus, vol. 5, Art. ID. 1066, 2016, doi: 10.1186/ s40064-016-2753-9

[13] H. L Dastjerdi and M. N. Ahmadabadi, "Moving least squares collocation method for Volterra integral equations with proportional delay", International journal of computer mathematics, vol. 94, no. 12, pp. 2335-2347, 2017, doi: 10.1080/ 00207160.2017.1283024

[14] E. Barkai, R. Metzler, and J. Klafter, "From continuous time random walks to the fractional Fokker-Planck equation", Physical review E, vol. 61, no. 1, pp. 132-138, 2000, doi: 10.1103/ physreve.61.132 
[15] D. A. Benson, S. W. Wheatcraft, and M M. Meerschaert, "Application of a fractional advection-dispersion equation". Water resources research, vol. 36, no. 6, pp. 1403-1412, 2000. [On line]. Available: https:/ / bit.ly/ 3xRoJve

[16] R. Hilfer, Ed., Applications of fractional calculus in physics. Singapore: World Scientific, 2000, doi: 10.1142/ 3779

[17] F. Mainardi, "Fractional calculus: some basic problems in continuum and statistical mechanics," in Fractals and fractional calculus in continuum mechanics, A. Carpinteri and F. Mainardi, Eds. Vienna: Springer, 1997, pp. 291-348, doi: 10.1007/978-3-7091-2664-6_7

[18] K. S. Miller and B. Ross, An introduction to the fractional calculus and fractional differential equations. New York, NY: John Wiley \& Sons, 1993.

[19] A. I. Saichev and G. M. Zaslavsky, "Fractional kinetic equations: solutions and applications", Chaos: an interdisciplinary journal of nonlinear science, vol. 7, no. 4, pp. 753-764, 1997, doi: 10.1063/ 1.166272

[20] H. Ding and C. Li, "Numerical algorithms for the fractional diffusionwave equation with reaction term", Abstract and applied analysis, vol. 2013, pp. Art ID. 493406, 2013, doi: 10.1155/ 2013/ 493406

[21] P. J. Torvik and R. L. Bagley, "On the appearance of the fractional derivative in the behavior of real materials", Journal of applied mechanics, vol. 51, no. 2, pp. 294-298, 1984, doi: 10.1115/ 1.3167615

[22] I. Podlubny, Fractional differential equations. San Diego, CA: Elsevier, 1999.

[23] T. M. Atanackovic and D. Zorica, "On the Bagley-Torvik equation", Journal of applied mechanics, vol. 80, no. 4, 2013, doi: $10.1115 / 1.4007850$

[24] S. Esmaeili and M. Shamsi, "A pseudo-spectral scheme for the approximate solution of a family of fractional differential equations", Communications in nonlinear science and numerical simulation, vol. 16, no. 9, pp. 3646-3654, 2011, doi.org: 10.1016/j.cnsns.2010.12.008

[25] Ş. Yüzbaşı, "Numerical solution of the Bagley-Torvik equation by the Bessel collocation method", Mathematical methods in the applied sciences, vol. 36, no. 3, pp. 300-312, 2012, doi: 10.1002/ mma.2588

[26] Y. Cenesiz, Y. Keskin, and A. Kurnaz, "The solution of the Bagley-Torvik equation with the generalized Taylor collocation method", Journal of the Franklin Institute, vol. 347, no. 2, pp. 452-466, 2010, doi: 10.1016/j.jfranklin.2009.10.007 
[27] T. Mekkaoui and Z Hammouch, "Approximate analytical solutions to the Bagley-Torvik equation by the fractional iteration method", Analele Universităţii din Craiova. Seria matematică, informatică (Online), vol. 39, no. 2, pp. 251-256, 2012.[On line]. Available: https:/ / bit.ly/ 3roy5fE

[28] K. Diethelm, J. Ford, "Numerical solution of the Bagley-Torvik equation", BIT numerical mathematics, vol. 42, pp. 490-507, 2002, doi: 10.1023/ A:1021973025166

[29] S. Mashayekhi and M. Razzaghi, "Numerical solution of the fractional Bagley-Torvik equation by using hybrid functions approximation", Mathematical methods in the applied sciences, vol. 39, no. 3, pp. 353-365, 2015, doi: 10.1002/ mma.3486 